\title{
ON A TAUBERIAN THEOREM OF WIENER AND PITT
}

\author{
HAROLD G. DIAMOND ${ }^{1}$
}

\begin{abstract}
N. Wiener and H. R. Pitt established a tauberian theorem which is "intermediate" between that of Wiener and Ikehara on one hand and a theorem of Hardy and Littlewood on the other. A new proof of the Wiener-Pitt theorem is given, using a technique of Bochner.
\end{abstract}

N. Wiener and H. R. Pitt established in [6] a tauberian theorem which is "intermediate" between that of Wiener and Ikehara on one hand and a theorem of Hardy and Littlewood on the other. Let $\alpha \in(0,1)$ and $B>0$. Let $\mathrm{C}=\mathcal{C}(\alpha, B)$ be the curve in the complex plane given by

$$
\left\{\sigma+i t:|t|=B \sigma^{\alpha}, 0 \leqq \sigma<\infty\right\} .
$$

From a one sided boundedness condition on a function plus an $L_{1}$ hypothesis upon its Laplace transform along the curve $\mathfrak{C}$, it is proved that the function has a small integral over certain intervals. A result of this type had been conjectured by J. Karamata and a special case $\left(\alpha=\frac{1}{2}\right)$ was given by V. G. Avakumović [1].

The proof of Wiener and Pitt was quite intricate. Another version appears in Pitt's book [3, pp. 135-138], but that proof is valid if and only if $\alpha \geqq \frac{1}{2}$. This is so because Pitt assumes that

$$
F(u):=\int_{-\infty}^{\infty} e^{i t u} \exp \left(-|t|^{1 / \alpha}\right) d t>0,
$$

and this inequality is valid for all real $u$ precisely when $\alpha \geqq \frac{1}{2}$ (cf. [2] and [4]). The object of the present paper is to give a shorter proof of the theorem. We shall use a Bochner type argument, integrating along line segments $\sigma+i t,-\lambda \leqq t \leqq \lambda$, where we take $\sigma=L / x, \lambda=\frac{1}{2} B^{\prime} \sigma^{\alpha}=$ $\frac{1}{2} B^{\prime} L^{\alpha} x^{-\alpha}$. The number $L$ will be chosen later and will be independent of $x$, and $B^{\prime}=\min (B, 1)$. We formulate the theorem substantially as in [3].

Received by the editors November 16, 1970 .

AMS 1970 subject classifications. Primary 40E05.

Key words and phrases. Tauberian theorem, Laplace transform, Wiener-Ikehara theorem.

${ }^{1}$ Research supported by grant NSF GP 12363. 
THEOREM. Let $s$ be a real measurable function that is supported in $(0, \infty)$, bounded from below, and satisfies $\int_{0}^{\infty}|s(y)| e^{-\sigma y} d y<\infty$ for all positive $\sigma$. For $\operatorname{Re} \omega>0$, define

and assume that

$$
S(\omega)=\int_{0}^{\infty} e^{-\omega y} s(y) d y
$$

$$
\lim _{\epsilon \rightarrow 0+} \int|S(\omega+\varepsilon)-S(\omega)||d \omega|=0,
$$

where the integration is taken over any bounded arc of $\mathrm{C}$. Then for any $A>0$,

$$
\lim _{x \rightarrow \infty} \frac{1}{2 A x^{\alpha}} \int_{x-A x^{\alpha}}^{x+A x^{\alpha}} s(y) d y=0 .
$$

Since $s$ has no support near zero, $S(\omega)$ vanishes exponentially as $\operatorname{Re} \omega \rightarrow$ $\infty$. This fact plus the $L_{1}$ limit hypothesis guarantee both the absolute integrability of $S$ along all of $\mathrm{C}$ and the validity of Cauchy's formula. Thus, for $|t| \leqq \lambda$ we can write

If we set

$$
S(\sigma+i t)=\frac{1}{2 \pi i} \int_{\mathrm{e}} \frac{S(z) d z}{z-\sigma-i t} .
$$

$$
h_{\lambda}(y)=\lambda\left(\frac{\sin \lambda y / 2}{\lambda y / 2}\right)^{2}
$$

we have the following familiar Parseval formula (cf. [5, p. 84])

$$
\int_{-\lambda}^{\lambda}\left(1-\frac{|t|}{\lambda}\right) e^{i t x} S(\sigma+i t) d t=\int_{0}^{\infty} h_{\lambda}(x-y) e^{-\sigma y} s(y) d y .
$$

Let $\varphi(x)$ denote the left-hand side of $\left(^{*}\right)$ (recalling that $\sigma$ and $\lambda$ are functions of $x$ ). We begin by establishing a type of Riemann-Lebesgue lemma.

LeMma 1.

$$
\lim _{x \rightarrow \infty} \varphi(x)=0 .
$$

Proof. Expressing $S(\sigma+i t)$ by the Cauchy formula and inverting the integration order, we have

$$
\varphi(x)=\frac{1}{2 \pi i} \int_{\mathrm{C}} S(z)\left\{\int_{-\lambda}^{\lambda} \frac{(1-|t| / \lambda)}{z-\sigma-i t} e^{i t x} d t\right\} d z .
$$

Let $\int_{-\lambda}^{\lambda}$ denote the inner integral, and integrate it by parts.

$$
\begin{aligned}
\left|\int_{-\lambda}^{\lambda}\right| & =\left|\int_{-\lambda}^{\lambda} \frac{e^{i t x}}{i x}\left\{\frac{(1-|t| / \lambda) i}{(z-\sigma-i t)^{2}}-\frac{\operatorname{sgn} t}{\lambda(z-\sigma-i t)}\right\} d t\right| \\
& \leqq \frac{1}{x} \int_{-\lambda}^{\lambda} \frac{d t}{|z-\sigma-i t|^{2}}+\frac{1}{\lambda x} \int_{-\lambda}^{\lambda} \frac{d t}{|z-\sigma-i t|} .
\end{aligned}
$$


Write $z=u+i v$. If $|v| \geqq \frac{3}{4} B^{\prime} \sigma^{\alpha}$, then

$$
|z-\sigma-i t| \geqq|\operatorname{Im}(z-\sigma-i t)| \geqq B^{\prime} \sigma^{\alpha} / 4
$$

and $\int_{-\lambda}^{\lambda}=o(1)$ as $x \rightarrow \infty$. If $|v|<\frac{3}{4} B^{\prime} \sigma^{\alpha}$, then $0 \leqq u<\left(3 B^{\prime} \mid 4\right)^{1 / \alpha} \sigma$ and

$$
|z-\sigma-i t|^{2}=(u-\sigma)^{2}+(v-t)^{2} \geqq c^{2} \sigma^{2}+(v-t)^{2},
$$

where $c^{2}=1-\left(3 B^{\prime} / 4\right)^{1 / \alpha}>0$. Thus, in this case

$$
\left|\int_{-\lambda}^{\lambda}\right| \leqq \frac{1}{x} \int_{-\infty}^{\infty} \frac{d t}{c^{2} \sigma^{2}+t^{2}}+\frac{1}{\lambda x} \frac{2 \lambda}{c \sigma}=O(1) \text {. }
$$

Now express $\int_{\mathcal{C}}$ as the integral over $\mathcal{C}_{1}=\left\{z \in \mathcal{C}:|\operatorname{Im} z|<\frac{3}{4} B^{\prime} \sigma^{\alpha}\right\}$ plus the integral over $\mathrm{C}_{2}=\mathrm{C}-\mathrm{C}_{1}$ and note that $\int_{\mathrm{C}_{1}}|S(z)||d z| \rightarrow 0$ as $1 / x$ (and hence $\sigma$ ) tends to 0 , while the same integral over $\mathrm{C}_{2}$ is uniformly bounded. Thus $\varphi(x) \rightarrow 0$ as $x \rightarrow \infty$

The next lemma shows $s$ to be bounded "on the average." It is essential for the estimation of $I_{2}$ and $I_{4}$ at the conclusion of the article that the number $\beta$ may be arbitrarily small and that the number $c_{1}$ not depend on $\beta$.

LEMma 2. There is a number $c_{1}$, which depends only on $s$, and for any number $\beta>0$ there is a number $X_{\beta}$, which increases with $1 / \beta$, such that, for all $x \geqq X_{\beta}$,

$$
\left(2 \beta x^{\alpha}\right)^{-1} \int_{x-\beta x^{\alpha}}^{x+\beta x^{\alpha}}|s(y)| d y \leqq c_{1} .
$$

Proof. By the hypothesis of the theorem, $s(y) \geqq-k$ for some $k$ and hence $|s(y)| \leqq s(y)+2 k$. We set $L=\left(2 / B^{\prime} \beta\right)^{1 / \alpha}$ (just for the proof of this lemma) and have $\sigma=L / x, \lambda=\frac{1}{2} B^{\prime} \sigma^{\alpha}=\left(\beta x^{\alpha}\right)^{-1}$. Then

$$
\begin{aligned}
& 2\left(\frac{\sin \frac{1}{2}}{\frac{1}{2}}\right)^{2} \exp \{-\sigma x-\sigma / \lambda\} \frac{\lambda}{2} \int_{x-1 / \lambda}^{x+1 / \lambda}|s(y)| d y \\
& \leqq \int_{0}^{\infty} h_{\lambda}(x-y) e^{-\sigma y}\{s(y)+2 k\} d y=\varphi(x)+2 k \int_{0}^{\infty} h_{\lambda}(x-y) e^{-\sigma y} d y \\
& \leqq|\varphi(x)|+2 k \exp \left\{-\sigma x+\lambda e^{L} / \lambda\right\} \int_{|y-x|<e^{L} / \lambda} h_{\lambda}(x-y) d y \\
& +2 k \int_{|y-x| \geqq e^{L / \lambda}} h_{\lambda}(x-y) d y \\
& \leqq|\varphi(x)|+2 k \exp \left\{-L+\beta L e^{L} x^{\alpha-1}\right\}+16 k e^{-L}<20 k e^{-L}
\end{aligned}
$$

provided that $x$ is large enough so that $|\varphi(x)| \leqq k e^{-L}, \exp \left\{\beta L e^{L} x^{\alpha-1}\right\} \leqq \frac{3}{2}$. Thus

$$
\begin{aligned}
\frac{\lambda}{2} \int_{x-1 / \lambda}^{x+1 / \lambda}|s(y)| d y & \leqq \frac{20 k}{8\left(\sin \frac{1}{2}\right)^{2}} \exp \left\{\beta L x^{\alpha-1}\right\} \\
& \leqq 12 k=: c_{1}
\end{aligned}
$$


provided $x$ is large enough so that $\exp \left\{\beta L x^{\alpha-1}\right\} \leqq \frac{24}{5}\left(\sin \frac{1}{2}\right)^{2}$.

Since $\beta L$ and $e^{L} \rightarrow \infty$ with $1 / \beta$ and $\varphi$ is continuous and tending to zero, we can take $X_{\beta}$ to be the infimum of all $x$ for which each of the three inequalities is valid.

COROLlary 1. There exists a number $c_{2}$, which depends only on $s$, such that, for all $x \geqq 0, \int_{0}^{x}|s(y)| d y \leqq c_{2} x$.

Proof. The hypotheses of the theorem imply that $|s|$ is locally integrable and that $s$ is zero near the origin. Now $x^{-1} \int_{0}^{x}|s(y)| d y$ is a continuous function on $(0, \infty)$ which is bounded at 0 and at $\infty$, and thus is bounded on $(0, \infty)$.

Corollary 2. Let $\beta>0$ and suppose that $w \geqq X_{\beta}$, where $X_{\beta}$ is as in the lemma. Suppose that $f$ is a positive monotone function on $[w, z]$ and $\frac{1}{2} \leqq f(x) / f\left(x+\beta x^{\alpha}\right) \leqq 2$ for all $x \in[w, z]$. If $z \geqq w+\beta w^{\alpha}$, then

$$
\int_{w}^{z} f(u)|s(u)| d u \leqq 4 c_{1} \int_{w}^{z} f(u) d u .
$$

Proof. We may assume without loss of generality that $f$ is increasing. If $z<\left(w+\beta w^{\alpha}\right)+\beta\left(w+\beta w^{\alpha}\right)^{\alpha}$, then

$$
\begin{aligned}
\int_{w}^{z} f(u)|s(u)| d u & \leqq f(z) \int_{w}^{z}|s(u)| d u \leqq 4 f(w) c_{1}(z-w) \\
& \leqq 4 c_{1} \int_{w}^{z} f(u) d u .
\end{aligned}
$$

In the other case define a sequence $\left\{x_{n}\right\}_{0}^{N+1}$ by taking $x_{0}=w, x_{n}=$ $x_{n-1}+\beta x_{n-1}^{\alpha}, n=1,2, \cdots$, and taking $x_{N+1}$ to be the largest number of the sequence not exceeding $z$. Apply the above inequalities to each of the intervals $\left[w, x_{1}\right],\left[x_{1}, x_{2}\right], \cdots,\left[x_{N}, z\right]$.

It is now convenient to approximate the right-hand side of $\left({ }^{*}\right)$ by a convolution. We show

LEMMA 3.

$$
\lim _{x \rightarrow \infty} \int_{0}^{\infty} h_{\lambda}(x-y) s(y) d y=0 .
$$

Proof. Let $\epsilon>0$ be given. Write

where

$$
\left(h_{\lambda} * s\right)(x)=\int_{0}^{\infty} h_{\lambda}(x-y) s(y) d y=\mathrm{I}+\mathrm{II},
$$

$$
\mathrm{I}=e^{L} \int_{0}^{\infty} h_{\lambda}(x-y) e^{-\sigma y} s(y) d y=e^{L} \varphi(x)=o(1)
$$


as $x \rightarrow \infty$ and

$$
\mathrm{II}=\int_{0}^{\infty} h_{\lambda}(x-y) s(y)\left\{1-e^{L-\sigma y}\right\} d y .
$$

Write

$$
\mathrm{II}=\int_{0}^{x / 2}+\int_{x / 2}^{x-v x^{\alpha}}+\int_{x-v x^{\alpha}}^{x+v x^{\alpha}}+\int_{x+v x^{\alpha}}^{\infty}
$$

where $v$ is a large positive number to be specified presently. By Corollary 1 ,

$$
\left|\int_{0}^{x / 2}\right| \leqq e^{L} \frac{4}{\lambda(x / 2)^{2}} \cdot c_{2} \frac{x}{2}=\frac{16 e^{L} c_{2}}{B^{\prime} L^{\alpha} x^{1-\alpha}}=o(1) .
$$

We can estimate the second and fourth integrals using Corollary 2. We first replace $h_{\lambda}(x-y)$ by $4 / \lambda(x-y)^{2}=: H(y)$, and note that $H$ satisfies the monotonicity and slow growth conditions of the corollary on each of the two ranges. Thus

$$
\left|\int_{x / 2}^{x-v x^{\alpha}}\right|+\left|\int_{x+v x^{\alpha}}^{\infty}\right| \leqq 2 \cdot 4 c_{1} e^{L} \frac{4}{\lambda \nu x^{\alpha}}<2 \epsilon / 5
$$

provided that $x$ is sufficiently large and $\nu>160 c_{1} e^{L} / B^{\prime} L^{\alpha} \epsilon$. With this choice of $\nu$, we estimate the third integral. If $x-v x^{\alpha} \leqq y \leqq x+v x^{\alpha}$ and $x$ is sufficiently large, then

$$
\left|e^{L-\sigma \nu}-1\right| \leqq 2|L-\sigma y| \leqq 2 L v x^{\alpha-1} .
$$

Thus, by Lemma 2, we have

$$
\left|\int_{x-v x^{\alpha}}^{x+v x^{\alpha}}\right| \leqq 2 L v x^{\alpha-1} \lambda \int_{x-v x^{\alpha}}^{x+v x^{\alpha}}|s(y)| d y=O\left(x^{\alpha-1} \lambda x^{\alpha}\right)=o(1) .
$$

Now if $x$ is sufficiently large, depending on $s, L, \mathrm{C}$ and $\epsilon$, we have $\left|\left(h_{\lambda} * s\right)(x)\right|<\epsilon$.

CONCLUSION OF THE ARGUMENT. Let $\chi_{E}$ be the indicator function of the set $E$. An easy estimate shows that for any positive number $M$ we have

$$
\begin{aligned}
h_{\lambda} * \chi_{[-M, M]}(u) & =1+O\left\{\lambda^{-1}(M-|u|)^{-1}\right\}, & & |u|<M, \\
& =O(1), & & \text { always, } \\
& =O\left\{M \lambda^{-1}(|u|-M)^{-2}\right\}, & & |u|>M .
\end{aligned}
$$

Here the constants implied by the $O$ 's are absolute. The preceding lemma implies that $\left((1 / 2 M) \chi_{[-M, M]} * h_{\lambda} * s\right)(x) \rightarrow 0$ as $x \rightarrow \infty$, where $M$ may tend to $\infty$ with $x$, so long as $x \geqq M$, say. We take $M=(A+\eta) x^{\alpha}$, 
where $\eta$ is a positive number, presently to be specified, and write

$$
\begin{aligned}
\left(\chi_{[-M, M]} * h_{\lambda} * s\right)(x)= & \int_{-\infty}^{x} s(x-y)\left(\chi_{[-M, M]} * h_{\lambda}\right)(y) d y \\
= & \int_{-\infty}^{-(A+2 \eta) x^{\alpha}}+\int_{-(A+2 \eta) x^{\alpha}}^{-A x^{\alpha}}+\int_{-A x^{\alpha}}^{A x^{\alpha}} \\
& +\int_{A x^{\alpha}}^{(A+2 \eta) x^{\alpha}}+\int_{(A+2 \eta) x^{\alpha}}^{x / 2}+\int_{x / 2}^{x} \\
= & \sum_{j=1}^{6} I_{j}, \text { say. }
\end{aligned}
$$

Now

$$
\mathrm{I}_{3}=\int_{x-A x^{\alpha}}^{x+A x^{\alpha}} s(y) d y+O\left(\frac{c_{1} A x^{\alpha}}{\lambda \eta x^{\alpha}}\right) .
$$

$\mathrm{I}_{2}+\mathrm{I}_{4}=O\left(c_{1} \eta x^{\alpha}\right), \quad \mathrm{I}_{1}+\mathrm{I}_{5}=O\left(c_{1} M / \lambda \eta x^{\alpha}\right)$, by Corollary 2 , and $\mathrm{I}_{6}=O\left(M c_{2} / \lambda x\right)$.

The four $O$ 's are absolute and the estimates are valid for all sufficiently large $x$. Thus we have

$$
\begin{gathered}
\left|\frac{1}{2 A x^{\alpha}} \int_{x-A x^{\alpha}}^{x+A x^{\alpha}} s(y) d y\right| \\
\leqq c_{3}\left\{\frac{c_{1}}{\eta B^{\prime} L^{\alpha}}+\frac{c_{1} \eta}{A}+\frac{c_{1}(A+\eta)}{A B^{\prime} L^{\alpha} \eta}+\frac{c_{2}(A+\eta) x^{\alpha-1}}{A B^{\prime} L^{\alpha}}+o(1)\right\},
\end{gathered}
$$

where $c_{3}$ is absolute.

Given $\epsilon$ in $(0,1)$, take $\eta$ so small that $c_{3} c_{1} \eta / A<\epsilon / 5$. Next, take $L$ sufficiently large that

$$
(A+\eta) c_{1} c_{3} /\left(A B^{\prime} L^{\alpha} \eta\right)<\epsilon / 5 .
$$

Then take $x$ so large that all the preceding inequalities are valid and

$$
c_{3} c_{2}(A+\eta) x^{\alpha-1} /\left(A B^{\prime} L^{\alpha}\right)+c_{3} o(1)<2 \epsilon / 5 .
$$

We conclude then that

$$
\left|\frac{1}{2 A x^{\alpha}} \int_{x-A x^{\alpha}}^{x+A x^{\alpha}} s(y) d y\right|<\epsilon
$$

for all sufficiently large $x$, and the proof of the theorem is complete.

Possibly the $L_{1}$ limit in the hypothesis of the theorem can be relaxed to the $L_{1}$ bound $\int_{\mathrm{C}}|S(\omega)||d \omega|<\infty$. One would then have to show (if possible) that the singularity of $S$ at zero, when approached from "within" $\mathcal{C}$, was sufficiently weak to permit the application of Cauchy's theorem. 


\section{REFERENCES}

1. V. G. Avakumović, Théorèmes relatifs aux intégrales de Laplace sur leur frontiêre de convergence, C. R. Acad. Sci. Paris 204 (1937), 224-226.

2. P. Lévy, Sur une application de la dérivée d'ordre non entier au calcul des probabilités, C. R. Acad. Sci. Paris 176 (1923), 1118-1120.

3. H. R. Pitt, Tauberian theorems, Tata Institute of Fundamental Research Monographs on Math. and Physics, no. 2, Oxford Univ. Press, London, 1958. MR 21 \#5109.

4. G. Pólya, On the zeros of an integral function represented by Fourier's integral, Messenger Math. 52 (1923), 185-188.

5. E. C. Titchmarsh, Introduction to the theory of Fourier integrals, Oxford Univ. Press, London, 1937.

6. N. Wiener and H. R. Pitt, A generalization of Ikehara's theorem, J. Math. Phys. 17 (1939), 247-258.

Department of Mathematics, University of Illinois, Urbana, Illinois 61801 